CARPATHIAN J. MATH.

Volume 38 (2022), No. 1,

Pages $149-158$
Online version at https : //www. carpathian. cunbm. utcluj. ro/

Print Edition: ISSN 1584 - 2851; Online Edition: ISSN 1843 - 4401

DOI: https://doi.org/10.37193/CJM.2022.01.12

Dedicated to the memory of Academician Mitrofan M. Choban (1942-2021)

\title{
Distinct partitions and overpartitions
}

\author{
MirCEA MERCA
}

ABSTRACT. In 1963, Peter Hagis, Jr. provided a Hardy-Ramanujan-Rademacher-type convergent series that can be used to compute an isolated value of the partition function $Q(n)$ which counts partitions of $n$ into distinct parts. Computing $Q(n)$ by this method requires arithmetic with very high-precision approximate real numbers and it is complicated. In this paper, we investigate new connections between partitions into distinct parts and overpartitions and obtain a surprising recurrence relation for the number of partitions of $n$ into distinct parts. By particularization of this relation, we derive two different linear recurrence relations for the partition function $Q(n)$. One of them involves the thrice square numbers and the other involves the generalized octagonal numbers. The recurrence relation involving the thrice square numbers provide a simple and fast computation of the value of $Q(n)$. This method uses only (large) integer arithmetic and it is simpler to program. Infinite families of linear inequalities involving partitions into distinct parts and overpartitions are introduced in this context.

\section{INTRODUCTION}

Recall [1] that a partition of a positive integer $n$ is a non-increasing sequence of positive integers whose sum is $n$. As usual, we denote by $p(n)$ the number of integer partitions of $n$ and we have the generating function

$$
\sum_{n=0}^{\infty} p(n) q^{n}=\frac{1}{(q ; q)_{\infty}} .
$$

Here and throughout this paper, we use the following customary $q$-series notation:

$$
\begin{aligned}
& (a ; q)_{n}= \begin{cases}1, & \text { for } n=0, \\
(1-a)(1-a q) \cdots\left(1-a q^{n-1}\right), & \text { for } n>0 ;\end{cases} \\
& (a ; q)_{\infty}=\lim _{n \rightarrow \infty}(a ; q)_{n} .
\end{aligned}
$$

Because the infinite product $(a ; q)_{\infty}$ diverges when $a \neq 0$ and $|q| \geqslant 1$, whenever $(a ; q)_{\infty}$ appears in a formula, we shall assume $|q|<1$.

A partition of the positive integer $n$ into distinct parts is a decreasing sequence of positive integers whose sum is $n$. As usual, we denote by $Q(n)$ the number of integer partitions of $n$ into distinct parts. For example, $Q(7)=5$ because the five partitions of 7 into distinct parts are

$$
(7),(6,1),(5,2),(4,3),(4,2,1) \text {. }
$$

We remark that the generating function of $Q(n)$ is given by

$$
\sum_{n=0}^{\infty} Q(n) q^{n}=(-q ; q)_{\infty}
$$

Received: 30.03.2021. In revised form: 17.10.2021. Accepted: 24.10.2021

2010 Mathematics Subject Classification. 11P81, 11P82, 05A19, 05A20 .

Key words and phrases. partitions, overpartitions, theta series, theta products . 
and the expansion starts as

$$
(-q ; q)_{\infty}=1+q+q^{2}+2 q^{3}+2 q^{4}+3 q^{5}+4 q^{6}+5 q^{7}+6 q^{8}+8 q^{9}+\cdots .
$$

We note that we often apply the Euler identity

$$
(-q ; q)_{\infty}=\frac{1}{\left(q ; q^{2}\right)_{\infty}} .
$$

There are well-known proofs of this identity. One of them is by Sylvester [35, pp. 13-14].

According to [45, eqs. 26.10.7, 26.10.8], the partition function $Q(n)$ satisfies the following linear recurrence relations:

$$
\sum_{k=-\infty}^{\infty}(-1)^{k} Q(n-k(3 k-1) / 2)= \begin{cases}(-1)^{m}, & \text { if } n=m(3 m-1), m \in \mathbb{Z} \\ 0, & \text { otherwise }\end{cases}
$$

and

$$
\sum_{k=-\infty}^{\infty}(-1)^{k} Q(n-k(3 k-1))= \begin{cases}1, & \text { if } n=m(m+1) / 2, m \in \mathbb{N}_{0} \\ 0, & \text { otherwise. }\end{cases}
$$

The number of terms in the first linear recurrence relation is about $\sqrt{8 n / 3}$, while in the second is about $\sqrt{4 n / 3}$. Due to E. Georgiadis, A. V. Sutherland and K. S. Kedlaya [47, A000009], we have a more efficient linear recurrence relation for $Q(n)$ in which the number of terms is about $\sqrt{n}$, i.e.,

$$
Q(n)+2 \sum_{k=1}^{\infty}(-1)^{k} Q\left(n-k^{2}\right)= \begin{cases}(-1)^{k}, & \text { if } n=m(3 m-1) / 2, m \in \mathbb{Z} \\ 0, & \text { otherwise. }\end{cases}
$$

The proofs of the linear recurrence relations (1.4)-(1.6), can be seen in [38, Corollaries 4.44.6]

The linear recurrence relation (1.6) with $Q(0)=1$ provides a simple and reasonably efficient way to compute the value of $Q(n)$. In fact, computing the value of $Q(n)$ with this recurrence relation requires all the values of $Q(k)$ with $k<n$.

There is a better way to compute an isolated value of $Q(n)$. P. Hagis [15-23] used the circle method to study various restricted partition functions. In [16], he provided a HardyRamanujan-Rademacher-type convergent series that can be used to compute an isolated value of the partition function $Q(n)$, i.e.,

$$
Q(n)=\frac{\pi}{\sqrt{24 n+1}} \sum_{\substack{k \geqslant 1 \\ k \text { odd }}} \frac{1}{k} \sum_{\substack{0 \leqslant h<k \\ \operatorname{gcd}(h, k)=1}} e^{-2 \pi n h / k} \frac{\omega(h, k)}{\omega(2 h, k)} I_{1}\left(\frac{\pi \sqrt{24 n+1}}{6 \sqrt{2} k}\right),
$$

where $\omega(h, k)$ is a $24 \mathrm{kth}$ root of unity that frequently occurs in the study of modular forms and is given by

$$
\omega(h, k)=\exp \left(\pi i \sum_{r=1}^{k-1} \frac{r}{k}\left(\frac{h r}{k}-\left\lfloor\frac{h r}{k}\right\rfloor-\frac{1}{2}\right)\right)
$$

and

$$
I_{\alpha}(z)=\sum_{r=0}^{\infty} \frac{1}{r ! \Gamma(\alpha+r+1)}\left(\frac{z}{2}\right)^{\alpha+2 r}
$$

is the modified Bessel function of purely imaginary argument. Computing $Q(n)$ by this formula requires arithmetic with very high-precision approximate real numbers and it is complicated. Details on how to efficiently implement a Hardy-Ramanujan-Rademacher type formula can be found in [26]. 
In this paper, we shall present a new linear recurrence relation for computing the value of $Q(n)$ which is more efficient than (1.6).

Theorem 1.1. For $n \geqslant 0$,

$$
Q(n)+2 \sum_{j=1}^{\infty}(-1)^{j} Q\left(n-3 j^{2}\right)= \begin{cases}1, & \text { if } n=m(3 m-1) / 2, m \in \mathbb{Z}, \\ 0, & \text { otherwise. }\end{cases}
$$

As we can see, the number of terms in this linear recurrence relation is about $\sqrt{n / 3}$. This allows us to calculate the value of $Q(n)$ in a way as simple as linear recurrence relation (1.6), but more efficiently. The algorithm uses only (large) integer arithmetic, and it is simpler to program.

We consider $\left(\rho_{n}\right)_{n \geqslant 0}$ to be a sequence of integers defined by

$$
\rho_{n}= \begin{cases}2 \cos \frac{(2 m+1) \pi}{3}, & \text { if } n=m(m+1) / 2, m \in \mathbb{N}_{0}, \\ 0, & \text { otherwise. }\end{cases}
$$

For any nonnegative integer $n$, it is clear that $\rho_{n} \in\{-2,0,1\}$. In addition, we remark that

$$
\begin{aligned}
\rho_{n(n+1) / 2} & = \begin{cases}-2, & \text { if } n \equiv 1 \quad(\bmod 3), \\
1, & \text { otherwise, }\end{cases} \\
\rho_{3 n} & = \begin{cases}1, & \text { if } n=m(3 m-1) / 2, m \in \mathbb{Z}, \\
0, & \text { otherwise, }\end{cases} \\
\rho_{3 n+1} & = \begin{cases}-2, & \text { if } n=3 m(m+1) / 2, m \in \mathbb{N}_{0}, \\
0, & \text { otherwise, }\end{cases} \\
\rho_{3 n+2} & =0 .
\end{aligned}
$$

We remark that Theorem 1.1 can be derived replacing $n$ by $3 n$ in the following surprising result.

Theorem 1.2. For $n \geqslant 0$,

$$
Q\left(\frac{n}{3}\right)+2 \sum_{j=1}^{\infty}(-1)^{j} Q\left(\frac{n}{3}-\frac{j^{2}}{3}\right)=\rho_{n},
$$

where $Q(x)=0$ if $x$ is not a nonnegative integer.

Moreover, replacing $n$ by $3 n+1$ in Theorem 1.2, we obtain a new linear recurrence relation for the partition function $Q(n)$.

Corollary 1.1. For $n \geqslant 0$,

$$
\sum_{j=-\infty}^{\infty}(-1)^{j} Q(n-j(3 j-2))= \begin{cases}1, & \text { if } n=3 m(m+1) / 2, m \in \mathbb{N}_{0} \\ 0, & \text { otherwise. }\end{cases}
$$

We note that this linear recurrence relation involves the general octagonal numbers $n(3 n-2), n \in \mathbb{Z}$ and is quite similar to (1.5) which involves twice generalized pentagonal numbers $n(3 n-1), n \in \mathbb{Z}$.

An overpartition of $n$ is a non-increasing sequence of natural numbers whose sum is $n$ in which the first occurrence of a number may be overlined [8]. Let $\bar{p}(n)$ denote the number of overpartitions of an integer $n$. For example, $\bar{p}(3)=8$ because there are 8 possible overpartitions of 3 : 
$(3),(\overline{3}),(2,1),(2, \overline{1}),(\overline{2}, 1),(\overline{2}, \overline{1}),(1,1,1),(\overline{1}, 1,1)$.

Since the overlined parts form a partition into distinct parts and the non-overlined parts form an ordinary partition, the generating function for overpartitions is given by

$$
\sum_{n=0}^{\infty} \bar{p}(n) q^{n}=\frac{(-q ; q)_{\infty}}{(q ; q)_{\infty}} .
$$

Overpartitions were introduced by S. Corteel and J. Lovejoy in [8] and have been the subject of many recent studies including G. E. Andrews [2], K. Bringmann and J. Lovejoy [5], W. Y. C. Chen and J. J. Y. Zhao [7], S. Corteel and P. Hitczenko [9], S. Corteel, W. M. Y. Goh and P. Hitczenko [10], S. Corteel and O. Mallet [11], A. M. Fu and A. Lascoux [12], M. D. Hirschhorn and J. A. Sellers [24,25], Kim [27], J. Lovejoy [29-34], K. Mahlburg [36], M. Merca [40,42] and A. V. Sills [46].

Recently, G. E. Andrews and M. Merca [4] introduced the overpartition function $\bar{M}_{k}(n)$, which counts the number of overpartitions of $n$ in which the first part larger than $k$ appears at least $k+1$ times. For example, $\bar{M}_{2}(12)=16$, and the partitions in question are:

$$
\begin{aligned}
& (4,4,4),(\overline{4}, 4,4),(3,3,3,3),(\overline{3}, 3,3,3),(3,3,3,2,1),(3,3,3, \overline{2}, 1), \\
& (3,3,3,2, \overline{1}),(3,3,3, \overline{2}, \overline{1}),(\overline{3}, 3,3,2,1),(\overline{3}, 3,3, \overline{2}, 1),(\overline{3}, 3,3,2, \overline{1}), \\
& (\overline{3}, 3,3, \overline{2}, \overline{1}),(3,3,3,1,1,1),(3,3,3, \overline{1}, 1,1),(\overline{3}, 3,3,1,1,1),(\overline{3}, 3,3, \overline{1}, 1,1) .
\end{aligned}
$$

The partition function $Q(n)$ and the overpartition function $\bar{M}_{k}(n)$ can be combined into an infinite family of identities as follows.

Theorem 1.3. For $n \geqslant 0$ and $k>0$,

$$
\begin{aligned}
& Q\left(\frac{n}{3}\right)+2 \sum_{j=1}^{k}(-1)^{j} Q\left(\frac{n}{3}-\frac{j^{2}}{3}\right) \\
& =\rho_{n}+(-1)^{k} \sum_{j=0}^{\infty} \rho_{j(j+1) / 2} \cdot \bar{M}_{k}(n-j(j+1) / 2),
\end{aligned}
$$

where $Q(x)=0$ if $x$ is not a nonnegative integer.

We remark that Theorem 1.2 is the limiting case $k \rightarrow \infty$ of Theorem 1.3. More explicitly, Theorem 1.3 can be rewritten as follows.

Corollary 1.2. For $n \geqslant 0$ and $k>0$,

$$
\begin{aligned}
& \text { i. } Q(n)+2 \sum_{j=1}^{\lfloor k / 3\rfloor}(-1)^{j} Q\left(n-3 j^{2}\right) \\
& =\rho_{3 n}+(-1)^{k} \sum_{j=0}^{\infty} \rho_{j(j+1) / 2} \cdot \bar{M}_{k}(3 n-j(j+1) / 2) \text {; } \\
& \text { ii. } \sum_{j=-\lfloor(k-1) / 3\rfloor}^{\lfloor(k+1) / 3\rfloor}(-1)^{j} Q(n-j(3 j-2)) \\
& =-\frac{\rho_{3 n+1}}{2}-(-1)^{k} \sum_{j=0}^{\infty} \frac{\rho_{j(j+1) / 2}}{2} \cdot \bar{M}_{k}(3 n+1-j(j+1) / 2) \text {; } \\
& \text { iii. } \sum_{j=0}^{\infty} \rho_{j(j+1) / 2} \cdot \bar{M}_{k}(3 n+2-j(j+1) / 2)=0 .
\end{aligned}
$$


Linear inequalities involving partition functions, especially Euler's partition function $p(n)$, have been the subject of recent studies $[3,4,14,28,37,38,41,43]$. Inspired by Corollary 1.2.i, we point out the following infinite families of linear inequalities.

Corollary 1.3. For $n \geqslant 0$ and $k>0$,

$$
\begin{aligned}
& \text { i. }(-1)^{k}\left(Q(n)+2 \sum_{j=1}^{k}(-1)^{j} Q\left(n-3 j^{2}\right)-\rho_{3 n}\right) \geqslant 0 \\
& \text { ii. }(-1)^{k+\lfloor k / 3\rfloor} \sum_{j=0}^{\infty} \rho_{j(j+1) / 2} \cdot \bar{M}_{k}(3 n-j(j+1) / 2) \geqslant 0 .
\end{aligned}
$$

Related to Corollary 1.2.ii, we remark that there is a substantial amount of numerical evidence to state the following conjecture involving the partition function $Q(n)$ and the generalized octogonal numbers $n(3 n-2), n \in \mathbb{Z}$.

Conjecture 1.1. For $n \geqslant 0$ and $k>0$,

$$
(-1)^{k}\left(\sum_{j=-(k-1)}^{k}(-1)^{j} Q(n-j(3 j-2))+\rho_{3 n+1} / 2\right) \leqslant 0 .
$$

For example,

$$
\begin{aligned}
& Q(n)-Q(n-1) \geqslant-\rho_{3 n+1} / 2, \\
& Q(n)-Q(n-1)-Q(n-5)+Q(n-8) \leqslant-\rho_{3 n+1} / 2, \\
& Q(n)-Q(n-1)-Q(n-5)+Q(n-8)+Q(n-16)-Q(n-21) \geqslant-\rho_{3 n+1} / 2 .
\end{aligned}
$$

It is clear that the general inequality provided by this conjecture can be restated in terms of the overpartition functions $\bar{M}_{k}(n)$ as follows: for $n \geqslant 0$ and $k>0$,

$$
(-1)^{k+\lceil k / 3\rceil} \sum_{j=0}^{\infty} \rho_{j(j+1) / 2} \cdot \bar{M}_{k}(3 n+1-j(j+1) / 2) \geqslant 0 .
$$

The rest of this paper is organized as follows. Considering a theta identity of Gauss and the Watson quintuple product identity, we will first prove Theorem 1.3 in Sect. 2. In Sect. 3 , we will provide a proof of Corollary 1.3. Other properties and open problems involving partitions into distinct parts and overpartitions will be remarked in the last section of the paper.

\section{PROOF OF THEOREM 1.3}

The following theta identity is often attributed to Gauss and sometimes Jacobi [1, p.23, eqs. (2.2.12)]:

$$
1+2 \sum_{n=1}^{\infty}(-1)^{n} q^{n^{2}}=\frac{(q ; q)_{\infty}}{(-q ; q)_{\infty}} .
$$

According to G. E. Andrews and M. Merca [4, Theorem 7], we have the following truncated version of (2.7):

$$
\begin{aligned}
& \frac{(-q ; q)_{\infty}}{(q ; q)_{\infty}}\left(1+2 \sum_{j=1}^{k}(-1)^{j} q^{j^{2}}\right) \\
& \quad=1+(-1)^{k} \frac{(-q ; q)_{k}}{(q ; q)_{k}} \sum_{j=k+1}^{\infty} \frac{2 q^{j(k+1)}}{1-q^{j}} \cdot \frac{\left(-q^{j+1} ; q\right)_{\infty}}{\left(q^{j+1} ; q\right)_{\infty}} .
\end{aligned}
$$


We remark that the series on the right hand side of this identity is the generating function for $\bar{M}_{k}(n)$, i.e.,

$$
\sum_{n=0}^{\infty} \bar{M}_{k}(n) q^{n}=\frac{(-q ; q)_{k}}{(q ; q)_{k}} \sum_{j=k+1}^{\infty} \frac{2 q^{j(k+1)}}{1-q^{j}} \cdot \frac{\left(-q^{j+1} ; q\right)_{\infty}}{\left(q^{j+1} ; q\right)_{\infty}} .
$$

The Watson quintuple product identity $[6,48]$ states that

$$
\sum_{n=-\infty}^{\infty} q^{n(3 n+1) / 2}\left(z^{-3 n}-z^{3 n+1}\right)=(z ; q)_{\infty}(q / z ; q)_{\infty}(q ; q)_{\infty}\left(q z^{2} ; q^{2}\right)_{\infty}\left(q / z^{2} ; q^{2}\right)_{\infty} .
$$

By this identity, with $q$ replaced by $q^{3}$ and $z$ replaced by $q$, we obtain

$$
\begin{aligned}
\sum_{n=-\infty}^{\infty} q^{3 n(3 n+1) / 2}\left(q^{-3 n}-q^{3 n+1}\right) & =\left(q ; q^{3}\right)_{\infty}\left(q^{2} ; q^{3}\right)_{\infty}\left(q^{3} ; q^{3}\right)_{\infty}\left(q^{5} ; q^{6}\right)_{\infty}\left(q ; q^{6}\right)_{\infty} \\
& =\frac{(q ; q)_{\infty}\left(q ; q^{2}\right)_{\infty}}{\left(q^{3} ; q^{6}\right)_{\infty}} \\
& =\frac{(q ; q)_{\infty}\left(-q^{3} ; q^{3}\right)_{\infty}}{(-q ; q)_{\infty}} .
\end{aligned}
$$

On the other hand, we have

$$
\begin{aligned}
\sum_{n=-\infty}^{\infty} q^{3 n(3 n+1) / 2}\left(q^{-3 n}-q^{3 n+1}\right) & =\sum_{n=-\infty}^{\infty} q^{3 n(3 n-1) / 2}-\sum_{n=-\infty}^{\infty} q^{(3 n+2)(3 n+1) / 2} \\
& =\sum_{\substack{n=0 \\
n \neq 1}}^{\infty} q^{n(n+1) / 2}-\sum_{\substack{(\bmod 3) \\
n=0}}^{\infty} 2 q^{n(n+1) / 2} \\
& =\sum_{n=0}^{\infty} \rho_{n(n+1) / 2} \cdot q^{n(n+1) / 2}
\end{aligned}
$$

Thus, we deduce that the generating function of $\rho_{n}$ is given by

$$
\sum_{n=0}^{\infty} \rho_{n} q^{n}=\frac{(q ; q)_{\infty}\left(-q^{3} ; q^{3}\right)_{\infty}}{(-q ; q)_{\infty}} .
$$

We can write

$$
\begin{aligned}
Q\left(\frac{n}{3}\right) & +2 \sum_{j=1}^{k}(-1)^{j} Q\left(\frac{n}{3}-\frac{j^{2}}{3}\right) \\
& =\left[q^{n}\right]\left(\left(-q^{3} ; q^{3}\right)_{\infty}\left(1+2 \sum_{j=1}^{k}(-1)^{j} q^{j^{2}}\right)\right) \\
& =\left[q^{n}\right]\left(\frac{(-q ; q)_{\infty}}{(q ; q)_{\infty}}\left(\sum_{n=0}^{\infty} \rho_{n} q^{n}\right)\left(1+2 \sum_{j=1}^{k}(-1)^{j} q^{j^{2}}\right)\right) \\
& =\left[q^{n}\right]\left(\left(\sum_{n=0}^{\infty} \rho_{n} q^{n}\right)\left(1+(-1)^{k} \sum_{n=0}^{\infty} \bar{M}_{k}(n)\right)\right) \\
& =\left[q^{n}\right]\left(\left(\sum_{n=0}^{\infty} \rho_{n} q^{n}\right)+(-1)^{k}\left(\sum_{n=0}^{\infty} \rho_{n} q^{n}\right)\left(\sum_{n=0}^{\infty} \bar{M}_{k}(n)\right)\right)
\end{aligned}
$$




$$
=\rho_{n}+(-1)^{k} \sum_{j=0}^{n} \rho_{j} \bar{M}_{k}(n-j) .
$$

This concludes the proof.

\section{Proof of Corollary 1.3}

Recently, M. Merca, C. Wang and A. J. Yee considered the identity (2.7) and provided in [44, Theorem 1.1] the following result: for $k>0$,

$$
\begin{aligned}
& \frac{\left(-q ; q^{2}\right)_{\infty}}{\left(q ; q^{2}\right)_{\infty}}\left(1+2 \sum_{j=1}^{k}(-1)^{j} q^{j^{2}}\right) \\
& =1+2 \sum_{j=1}^{\infty}(-1)^{j} q^{2 j^{2}}+(-1)^{k} 2 q^{(k+1)^{2}}\left(-q ; q^{2}\right)_{\infty} \sum_{j=0}^{\infty} \frac{q^{(2 k+2 j+3) j}}{\left(q^{2} ; q^{2}\right)_{j}\left(q ; q^{2}\right)_{k+j+1}} .
\end{aligned}
$$

This identity can be written as

$$
\begin{aligned}
&(-q ; q)_{\infty}\left(1+2 \sum_{j=1}^{k}(-1)^{j} q^{j^{2}}\right) \\
&=\frac{\left(q^{2} ; q^{2}\right)_{\infty}}{\left(-q ; q^{2}\right)_{\infty}\left(-q^{2} ; q^{2}\right)_{\infty}}+(-1)^{k} 2 q^{(k+1)^{2}} \sum_{j=0}^{\infty} \frac{q^{(2 k+2 j+3) j}}{\left(q^{2} ; q^{2}\right)_{j}\left(q ; q^{2}\right)_{k+j+1}} \\
&=(q ; q)_{\infty}+(-1)^{k} 2 q^{(k+1)^{2}} \sum_{j=0}^{\infty} \frac{q^{(2 k+2 j+3) j}}{\left(q^{2} ; q^{2}\right)_{j}\left(q ; q^{2}\right)_{k+j+1}} .
\end{aligned}
$$

By this identity, with $q$ replaced by $q^{3}$, we get

$$
\begin{aligned}
& \left(-q^{3} ; q^{3}\right)_{\infty}\left(1+2 \sum_{j=1}^{k}(-1)^{j} q^{3 j^{2}}\right) \\
& \quad=\left(q^{3} ; q^{3}\right)_{\infty}+(-1)^{k} 2 q^{3(k+1)^{2}} \sum_{j=0}^{\infty} \frac{q^{3 j(2 k+2 j+3)}}{\left(q^{6} ; q^{6}\right)_{j}\left(q^{3} ; q^{6}\right)_{k+j+1}}
\end{aligned}
$$

Multiplying both sides of (3.10) by

$$
\frac{(-q ; q)_{\infty}}{\left(-q^{3} ; q^{3}\right)_{\infty}}
$$

we obtain

$$
\begin{aligned}
& (-q ; q)_{\infty}\left(1+2 \sum_{j=1}^{k}(-1)^{j} q^{3 j^{2}}\right) \\
& =\left(-q ; q^{3}\right)_{\infty}\left(-q^{2} ; q^{3}\right)_{\infty}\left(q^{3} ; q^{3}\right)_{\infty}+\frac{(-1)^{k} 2 q^{3(k+1)^{2}}}{\left(q ; q^{6}\right)_{\infty}\left(q^{5} ; q^{6}\right)_{\infty}} \sum_{j=0}^{\infty} \frac{q^{3 j(2 k+2 j+3)}}{\left(q^{6} ; q^{6}\right)_{j}\left(q^{3} ; q^{6}\right)_{k+j+1}} \\
& =\sum_{j=-\infty}^{\infty} q^{j(3 j-1) / 2}+\frac{(-1)^{k} 2 q^{3(k+1)^{2}}}{\left(q ; q^{6}\right)_{\infty}\left(q^{5} ; q^{6}\right)_{\infty}} \sum_{j=0}^{\infty} \frac{q^{3 j(2 k+2 j+3)}}{\left(q^{6} ; q^{6}\right)_{j}\left(q^{3} ; q^{6}\right)_{k+j+1}},
\end{aligned}
$$


where we have invoked the Jacobi triple product identity (cf. [13, Eq. (1.6.1)])

$$
(z ; q)_{\infty}(q / z ; q)_{\infty}(q ; q)_{\infty}=\sum_{n=-\infty}^{\infty}(-z)^{n} q^{n(n-1) / 2}
$$

with $q$ replaced by $q^{3}$ and $z$ replaced by $-q$.

Considering that the coefficients of $q^{n}$ in the expansion

$$
\frac{q^{3(k+1)^{2}}}{\left(q ; q^{6}\right)_{\infty}\left(q^{5} ; q^{6}\right)_{\infty}} \sum_{j=0}^{\infty} \frac{q^{3 j(2 k+2 j+3)}}{\left(q^{6} ; q^{6}\right)_{j}\left(q^{3} ; q^{6}\right)_{k+j+1}}
$$

are all non-negatives, we deduce

$$
\begin{aligned}
(-1)^{k} & \left(Q(n)+2 \sum_{j=1}^{k}(-1)^{j} Q\left(n-3 j^{2}\right)-\rho_{3 n}\right) \\
& =\left[q^{n}\right](-1)^{k}\left((-q ; q)_{\infty}\left(1+2 \sum_{j=1}^{k}(-1)^{j} q^{3 j^{2}}\right)-\sum_{j=-\infty}^{\infty} q^{j(3 j-1) / 2}\right) \\
& =\left[q^{n}\right]\left(\frac{2 q^{3(k+1)^{2}}}{\left(q ; q^{6}\right)_{\infty}\left(q^{5} ; q^{6}\right)_{\infty}} \sum_{j=0}^{\infty} \frac{q^{3 j(2 k+2 j+3)}}{\left(q^{6} ; q^{6}\right)_{j}\left(q^{3} ; q^{6}\right)_{k+j+1}}\right) \\
& \geqslant 0 .
\end{aligned}
$$

The second identity follows easily considering Corollary 1.2.i.

\section{CONCLUDING REMARKS AND OPEN PROBLEMS}

Considering a theta identity of Gauss and a special case of the Watson quintuple product identity, we obtain a recurrence relation for the number of distinct partitions of $n$ which combines two different recurrence relations into one. The first recurrence relation for the partition function $Q(n)$ involves the thrice square numbers and the second involves the octagonal numbers.

Taking into account Euler's pentagonal number theorem

$$
\sum_{n=-\infty}^{\infty}(-1)^{n} q^{n(3 n-1) / 2}=(q ; q)_{\infty}
$$

and the identity

$$
(-q ; q)_{\infty}=\frac{(-q ; q)_{\infty}}{(q ; q)_{\infty}} \cdot(q ; q)_{\infty}
$$

we easily deduce that the partition function $Q(n)$ is closely related to overpartition function $\bar{p}(n)$, i.e.,

$$
Q(n)=\sum_{j=-\infty}^{\infty}(-1)^{j} \bar{p}(n-j(3 j-1) / 2) .
$$

Rewriting the generating function (2.9) as

$$
\left(-q^{3} ; q^{3}\right)_{\infty}=\frac{(-q ; q)_{\infty}}{(q ; q)_{\infty}} \sum_{n=0}^{\infty} \rho_{n(n+1) / 2} \cdot q^{n(n+1) / 2},
$$

we easily derive a new decomposition of $Q(n)$ in terms of the overpartition function $\bar{p}(n)$ and a new linear homogeneous recurrence relation for $\bar{p}(n)$ when $n$ in not divisible by 3 . 
Corollary 4.4. For $n \geqslant 0$,

$$
Q(n)=\sum_{j=0}^{\infty} \rho_{j(j+1) / 2} \cdot \bar{p}(3 n-j(j+1) / 2) .
$$

Corollary 4.5. For $n \not \equiv 0(\bmod 3)$,

$$
\sum_{j=0}^{\infty} \rho_{j(j+1) / 2} \cdot \bar{p}(n-j(j+1) / 2)=0 .
$$

Inspired by these corollaries, we experimentally discovered the following conjectures involving the overpartition function $\bar{p}(n)$ and the partition function $Q(n)$.

Conjecture 4.2. For $n, k \geqslant 0$,

$$
\sum_{j=0}^{3 k+2} \rho_{j(j+1) / 2} \cdot \bar{p}(n-j(j+1) / 2) \leqslant \begin{cases}Q(n / 3), & \text { if } n \equiv 0 \quad(\bmod 3) \\ 0, & \text { otherwise, }\end{cases}
$$

with strict inequality if and only if $n \geqslant(3 k+3)(3 k+4) / 2$.

Conjecture 4.3. For $n, k \geqslant 0$,

$$
\begin{gathered}
\bar{p}(n-(3 k+1)(3 k+2) / 2)+\sum_{j=0}^{3 k+1} \rho_{j(j+1) / 2} \cdot \bar{p}(n-j(j+1) / 2) \\
\geqslant \begin{cases}Q(n / 3), & \text { if } n \equiv 0 \quad(\bmod 3) \\
0, & \text { otherwise, }\end{cases}
\end{gathered}
$$

with strict inequality if and only if $n \geqslant(3 k+1)(3 k+2) / 2$.

\section{REFERENCES}

[1] Andrews, G. E. The Theory of Partitions. Cambridge Mathematical Library, Cambridge University Press, Cambridge, 1998. Reprint of the 1976 original.

[2] Andrews, G. E. Singular overpartitions. Int. J. Number Theory 11 (2015), no. 5, 1523-1533.

[3] Andrews, G. E.; Merca, M. The truncated pentagonal number theorem. J. Combin. Theory Ser. A 119 (2012), 1639-1643.

[4] Andrews, G. E.; Merca, M. Truncated theta series and a problem of Guo and Zeng. J. Combin. Theory Ser. A 154 (2018), 610-619.

[5] Bringmann, K.; Lovejoy, J. Dyson's rank, overpartitions, and weak Maass forms. Int. Math. Res. Not. IMRN (2007), rnm063.

[6] Carlitz, L.; Subbarao, M. V. A simple proof of the quintuple product identity. Proc. Amer. Math. Soc. 32 (1972), 42-44.

[7] Chen, W. Y. C.; Zhao, J. J. Y. The Gaussian coefficients and overpartitions. Discrete Math. 305 (2005), $350-353$.

[8] Corteel, S.; Lovejoy, J. Overpartitions. Trans. Amer. Math. Soc. 356 (2004), 1623-1635.

[9] Corteel, S.; Hitczenko, P. Multiplicity and number of parts in overpartitions. Ann. Comb. 8 (2004), no. 3, 287-301.

[10] Corteel, S.; Goh, W. M. Y.; Hitczenko, P. A local limit theorem in the theory of overpartitions. Algorithmica 46 (2006), 329-343.

[11] Corteel, S.; Mallet, O. Overpartitions, lattice paths, and Rogers-Ramanujan identities. J. Combin. Theory Ser. A 114 (2007), no. 8, 1407-1437.

[12] Fu, A. M.; Lascoux, A. q-identities related to overpartitions and divisor functions. Electron. J. Combin. 12 (2005), RP38.

[13] Gasper, G.; Rahman, M. Basic Hypergeometric Series. Encyclopedia of Mathematics And Its Applications 35, Cambridge University Press, Cambridge, 1990.

[14] Guo, V. J. W.; Zeng, J. Two truncated identities of Gauss. J. Combin. Theory Ser. A 120 (2013), 700-707.

[15] Hagis, P. A problem on partitions with a prime modulus $p \geqslant 3$. Trans. Am. Math. Soc. 102 (1962), 30-62.

[16] Hagis, P. Partitions into odd summands. Am. J. Math. 85 (1963), 213-222.

[17] Hagis, P. On a class of partitions with distinct summands. Trans. Am. Math. Soc. 112 (1964), 401-415. 
[18] Hagis, P. Partitions into odd and unequal parts. Am. J. Math. 86 (1964), 317-324.

[19] Hagis, P. Partitions with odd summands-some comments and corrections. Am. J. Math. 87 (1965), 218-220.

[20] Hagis, P. A correction of some theorems on partitions. Trans. Am. Math. Soc. 118 (1965), 550.

[21] Hagis, P. On partitions of an integer into distinct odd summands. Am. J. Math. 87 (1965), 867-873.

[22] Hagis, P. Some theorems concerning partitions into odd summands. Am. J. Math. 88 (1966), 664-681.

[23] Hagis, P. Partitions with a restriction on the multiplicity of summands. Trans. Am. Math. Soc. 155 (1971), 375-384.

[24] Hirschhorn, M. D.; Sellers, J. A. Arithmetic relations for overpartitions J. Combin. Math. Combin. Comp. 53 (2005), 65-73.

[25] Hirschhorn, M. D.; Sellers, J. A. Arithmetic properties of overpartitions into odd parts. Ann. Comb. 10 (2006), no. 3, 353-367.

[26] Johansson, F. Efficient implementation of the Hardy-Ramanujan-Rademacher formula. LMS J. Comput. Math. 15 (2012), no. 1, 341-359.

[27] Kim, B. A short note on the overpartition function. Discrete Math. 309 (2009), 2528-2532.

[28] Katriel, J. Asymptotically trivial linear homogeneous partition inequalities J. Number Theory 184 (2018), $107-121$.

[29] Lovejoy, J. Gordon's theorem for overpartitions. J. Comb. Theory Ser. A 103 (2003), 393-401.

[30] Lovejoy, J. Overpartition theorems of the Rogers-Ramanujan type. J. London Math. Soc. 69 (2004), 562-574.

[31] Lovejoy, J. Overpartitions and real quadratic fields. J. Number Theory 106 (2004), 178-186.

[32] Lovejoy, J. A theorem on seven-colored overpartitions and its applications. Int. J. Number Theory 1 (2005), $215-224$.

[33] Lovejoy, J. Rank and conjugation for the Frobenius representation of an overpartition. Ann. Comb. 9 (2005), 321-334.

[34] Lovejoy, J. Partitions and overpartitions with attached parts. Arch. Math. (Basel) 88 (2007), 316-322.

[35] MacMahon,P. A.Combinatory Analysis, Chelsea Publishing Company, New York, NY, Vol. 2, 1984. Originally published in two volumes at Cambridge, 1918. Published at New York as two volumes in one, 1984.

[36] Mahlburg, K. The overpartition function modulo small powers of 2. Discrete Math. 286 (2004), 263-267.

[37] Merca, M. Fast algorithm for generating ascending compositions. J. Math. Model. Algorithms 11 (2012), 89-104.

[38] Merca, M. A new look on the truncated pentagonal number theorem. Carpathian J. Math. 32 (2016), 97-101.

[39] Merca, M. Combinatorial interpretations of a recent convolution for the number of divisors of a positive integer. J. Number Theory 160 (2016), 60-75.

[40] Merca, M. From a Rogers's identity to overpartitions. Period. Math. Hungar. 75 (2017), 172-179.

[41] Merca, M. Polygonal numbers and Rogers-Ramanujan-Gordon theorem. Ramanujan J. 55 (2021), 783-792.

[42] Merca, M. On a nonlinear relation for computing the overpartition function. Math. Slovaca, 71 (2021), $535-542$.

[43] Merca, M.; Katriel, J. A general method for proving the non-trivial linear homogeneous partition inequalities. Ramanujan J. 51 (2020), no. 2, 245-266.

[44] Merca, M.; Wang, C.; Yee, A. J. A truncated theta identity of Gauss and overpartitions into odd parts. Ann. Comb., 23 (2019), 907-915.

[45] Olver, F. W. J.; Lozier, D. W.; Boisvert, R. F.; Clark, C. W. NIST Handbook of Mathematical Functions. Cambridge University Press, Cambridge, 2010.

[46] Sills, A. V. A Rademacher Type Formula for Partitions and Overpartitions, Int. J. Math. Math. Sci. (2010), Article ID 630458.

[47] Sloane, N. J. A. The on-line encyclopedia of integer sequences. Published electronically at http: / / oeis . org 2021.

[48] Subbarao, M .V.; Vidyasagar, M. On Watson's quintuple product identity. Proc. Amer. Math. Soc. 26 (1970), 23-27.

\author{
DEPARTMENT OF MATHEMATICS \\ UNIVERSITY OF CRAIOVA \\ 200585 CRAIOVA, ROMANIA \\ Email address: mircea.merca@profinfo.edu.ro
}

